\title{
Tobacco use and the quality of life
}

The paper by Lyons, Lo, and Littlepage in this issue of Tobacco Control ${ }^{1}$ makes important contributions to two fields that now can be joined, health status assessment and prevention of tobacco use.

In their paper, the authors demonstrated statistically significant and clinically substantial decrements among ever-smokers in four quality-of-life indicators that are at the physical health end of the health status profile. Eversmokers, compared to never-smokers, ascribed to themselves lower ratings on physical functioning, experience of pain, general health perception, and vitality. In common terms, ever-smokers reported limitations in performing physical activities such as carrying packages and climbing stairs, the frequent experience of pain that sometimes interferes with work, and feeling tired and worn out more of the time; ever-smokers have less confidence in their health, believe that their health is not as good as that of other people they know, and expect their health is going to get worse. There were no decrements in functioning or well-being at the opposite or mental health end of the SF36 profile on self-perceptions of mental health, limitations at work due to emotional difficulties, and social functioning.

The analytic strategy that the authors employed adjusted for differences in age, sex, alcohol intake, and social class, major confounders known to influence health status assessment. ${ }^{2,3}$ Disease and disease severity were not adjusted for because the sample was drawn systematically from a general population where the prevalence of disease and impairment unassociated with smoking could be expected to be low and equal in the two groups. Therefore, we can believe that smoking itself exerts an independent negative effect on an individual's quality of life. Conventional thought holds that most smokers began in early adolescence largely out of peer pressure and a desire to appear more mature and to fit in. Those motivations dissipate in early adulthood. Why then do adults continue to smoke even though they are aware that smoking diminishes their quality of life, is a major source of suffering, and is likely to shorten their life by about six years? Addiction, in the real sense, must be the answer.

In addition to being the first to report on the effects of smoking on functional status and well-being, Lyons, Lo, and Littlepage introduced a novel method for calibrating their data in easily understood terms. It was already known $^{3}$, and Lyons and colleagues confirmed, that scores on concepts at the physical end of the SF-36 profile decline with age, whereas the scores on the mental health end tend to remain level or to rise. Using the decrements normally expected of a year of aging in a normal population as the denominator, and the actual decrements observed in eversmokers as the numerator, they calculated the age equivalency of the effect of smoking (table).

The age equivalency method is a more dramatic and personally interpretable way of conveying to the public the
Table Effects of smoking on four concepts of health

\begin{tabular}{ll}
\hline Health concept & $\begin{array}{l}\text { Ever-smokers' scores are } \\
\text { equivalent to never-smokers who are }\end{array}$ \\
\hline Physical functioning & 6.6 years older \\
Pain & 15.6 years older \\
General health perception & 14.6 years older \\
Vitality & 14.0 years older \\
\hline
\end{tabular}

Source: Lyons, Lo, and Littlepage 5.6 years olde 14.0 years older

ravaging effects of tobacco smoking than use of the raw scores alone. The authors express an interest in primary prevention. The well-known adverse effects of smoking on breath quality, illness, absenteeism, level of productivity, and death have seemed to me too remote to adolescent interests to be maximally effective. The age-equivalency method gets closer to those interests and deserves a try in school-based health education programmes. Starfield ${ }^{4}$ has developed an adolescent health status measure, and Landgraf and Ware (personal communication) plan to release later in 1994 a short-form health status survey for ages 5 to 15 years that will be distributed without charge by the Medical Outcomes Trust (Boston, Massachusetts, USA). Concepts of health relevant to adolescents are captured in those instruments. They might reveal among adolescent smokers impairments in health domains that are closer to their motivational core.

Cigarette smoking is the developed world's major health risk factor. The US Centers for Disease Control and Prevention have estimated, using smoking-attributable mortality calculations, that tobacco accounted for $19.4 \%$ of all deaths in the US in $1990 .^{5}$ Thus, tobacco smoking accounted for an average of 1147 deaths per day. Tobacco is the OECD nations' premier toxin with respect to deaths, years of potential life lost, illness, disability, and now, through the work of Lyons et al, quality-of-life years wasted.

Medical Outcomes Trust,

ALVIN R TARLOV

750 Washington Street,

Boston, Massachusetts 02111, USA

1 Lyons RA, Lo SV, Littlepage BNC. Perception of health amongst eversmokers and never smokers: a comparison using the SF-36 health survey questionnaire. Tobacco Control 1994; 3: 213-5

2 Tarlov AR, Ware JE Jr, Greenfield S, et al. The Medical Outcomes study: an application of methods for monitoring the results of medical care. fAMA 1989; 262: 925-30.

3 Ware JE Jr, Snow, KK, Kosinski M, Gandek B. SF-36 health survey, manual and interpretation guide, 1993 . Boston, Massachusetts: The

4 Starfield B, Bergner M, Ensminger $M$, et al. Adolescent health status measurement: development of the child health and illness profile. Pediatrics 1993; 91: 430-5.

5 US Centers for Disease Control and Prevention. Cigarette smokingAttributable mortality and years of potential life lost - United States,
1990. MMWR 1993; 42: 645-9. 